\title{
Role of biosurfactants in nature and biotechnological applications
}

\begin{abstract}
In nature, biosurfactants are amphiphilic molecules, capable to emulsify and decrease surface tension water, mainly glycolipids are the most known and studied produced by Pseudomonas aeruginosa. These compounds are produced by microorganisms (bacteria, actinomycetes and fungi) who has genes, metabolic pathways and as a consequence are physiologically adapted in order to uptake organic matter non-soluble. Recently, the interest for its biotechnological production has increased, because of their environmental, industrial, and pharmaceutical applications, and others. Through microbial biodiversity exploration, on terrestrial or aquatic environments polluted with persistant organic compounds non-soluble in water, is possible to isolate microorganisms with tensoactive properties and capable to biodegrade pollutants. This can be done by use of selective culture media, tensoactive tests and molecular tools. Biosurfactants are promising products and actually some commercial products are applied and patented.
\end{abstract}

Keywords: glycolipids, surface tension, rhamnolipids, emulsifiers
Volume 2 Issue 4 - 2016

\author{
Gustavo Yañez Ocampo \\ Laboratory of Edaphology and Environment, Autonomous \\ University of the State of México, México
}

\begin{abstract}
Correspondence: Gustavo Yañez Ocampo, Laboratory of Edaphology and Environment, Faculty of Sciences, Autonomous University of the State of México, Instituto Literario \# 100, CP $50000 \mathrm{Col}$ Centro,Toluca, State of México, México, Tel (01722) 2262300,Email yanezg0206@gmail.com
\end{abstract}

Received:September 03, 2016 | Published: September 07 2016

\section{Introduction}

The bioavailability of organic compounds, commonly is ruled by physical-chemical processes such as sorption and desorption, diffusion and dissolution. Some microorganisms improve bioavailability of biodegradable organic matter by production of biosurfactants. Biosurfactants (BS) are exopolymers mainly produced as secondary metabolites by bacteria, yeasts and fungi. By their molecular weight can be classified as BS of low molecular weight, such as glycolipids and lipopeptides; they are more effective at lowering the interfacial and surface tensions (reduce water surface tension from 72 to $25 \mathrm{mN} / \mathrm{m}$ approximately), and BS of high molecular weight, polysaccharides, lipoproteins and lipopolysaccharides; they are effective stabilizers of oil-in-water emulsions, also have emulsificant and dispersant properties.

Biochemically, BS are amphiphilic biomolecules, with hydrophobic and hydrophilic portions, the hydrophobic part of the molecule is based on saturated or unsaturated fatty acids, the hydrophilic portion can be either cationic and anionic amino acids or mono-and di-saccharides (rhamnose and trehalose respectively). Both portions can have a wide range of chemical structural features. ${ }^{1}$ The best-studied microbial surfactants are glycolipids, among these, the best-known compounds are rhamnolipids, trehalolipids, sophorolipids and mannosylerythritol lipids. The BS most studied and better known are rhamnolipids produced by Pseudomonas species, trehalolipids produced by Rhodococcus species, surfactin by Bacillus subtilis, sophorolipids by Candida bombicola ${ }^{2}$ and emulsan by Acinetobacter calcoaceticus. $^{3}$

\section{Role of biosurfactants in nature}

In soil, water or sediments, the microbiota has a lot synergistics or anthagonistics relationships. By example some bacteria have to compete with others for space or resources, therefore, in order to colonize these environments, bacteria have a quorum sensing system, is a communication system characterized by the secretion and detection of signal molecules within a bacterial population. Biosurfactants (BS) are synthesized in response to an ecological strategy to survive under different physical and chemical stresses mediated by a specific quorum sensing system regulated by environmental or nutrimental conditions, often is also activated in high cell densities, so it represents a direct link between high cell population and rhamnolipid production activation.

The rhamnolipids produced by Pseudomonas aeruginosa have a quorum sensing system regulated by limitation of nitrogen. The synthesis of mono and dirhamnolipids involves rhamnosyltransferases RhlB y RhlC mainly, and is strongly controlled by genetic regulation (transcriptional and postranscriptional) of the rmlBDAC and rhlAB operons. A metabolic network has been proposed by SoberónChávez et al., ${ }^{4}$ briefly, synthesis of L-rhamnose derives either from gluconeogenesis or the Entner-Doudoroff-Pathway and the synthesis of hydrophobic $\beta$-hydroxyalkanoic acid moiety originates directly from fatty acid de novo synthesis system.

In general BS, and specifically rhamnolipids, have different physiological functions such as uptake of poorly accessible substrates like hydrophobic molecules due to their tensoactive properties. This is particulary useful, for competing with other microorganism due to their wide range of antimicrobial activity and the adhesion or contact to hydrophobic surfaces.

In several environments like sea, mangrove sediments or soil polluted with oil hydrocarbon or pesticides, the microorganisms produce biosurfactants, mainly when they are exposed to low solubility carbon sources. ${ }^{5}$ In vitro conditions, is known that an excess in carbon source (by example glucose, glicerol or frying oils used) and stress with low levels of nitrogen, promotes rhamnolipids production. ${ }^{6}$ 


\section{Biotechnological biosurfactants}

\section{applications}

The microbial biosurfactants can enhance bioavailability and biodegradability of low solubility compounds through reducing surface and interfacial tension at gas-liquid-solid interfaces, also have emulsifying and foaming properties. ${ }^{7}$ In comparison with synthetic surfactants, BS are more biodegradable and less toxics, for this reason they have been applied in bioremediation processes of polluted environmental sites with heavy metals, organophosphate pesticides (lubricants, surfactant aided bioremediation) and hydrocarbon total petroleum (enhanced oil recovery, crude oil drilling). ${ }^{8}$ In addition, BS have applications in the alimentary, pharmaceutical and cosmetical industry (health care and food processing). ${ }^{9}$

In the quest for biotechnological production of BS, is necessary to explore microbial biodiversity of environments in order to isolate and identify microbial strains potentially producers of BS, by using selective and differential media, by molecular tools and ensuring their activity by assays of emulsion, tensoactive, hemolytic and dispersant activity. ${ }^{9}$ In addition, it is required the research of the optimal culture conditions in liquid medium such as $\mathrm{pH}$, agitation speed, temperature and selection of the carbon source for the culture medium. In this sense, nowadays there is an interest for using low cost carbon sources such as soja oil, corn oil used, glycerol, lacto serum, coffee waste, and others hydrophobic compounds by example n-decane, n-tetradecane, paraffin..$^{10}$ Fortunately, BS are extracellular products, so is relatively simple to separate and collect them from the culture medium.

BS are promising bioproducts Shete et al., ${ }^{11}$ reported the patents on biosurfactants and bioemulsifiers (255 patents issued worldwide) showing high number of patents in the petroleum industry $(33 \%)$, cosmetics (15\%), antimicrobial agent and medicine $(12 \%)$ and bioremediation (11\%). Sophorolipids (24\%), surfactin $(13 \%)$ and rhamnolipids (12\%).

\section{Conclusion}

The tensoactive properties of biosurfactants in nature like glycolipids, have specific functions in the environment. From this knowledge, biotechnology it has dedicated to isolate microorganisms biosurfactant producers, in order to give them new applications such as lubricants, wetting, softening, fixing dyes, emulsion makers, foaming, as well as in food, biomedical and pharmaceutical industry, and bioremediation of organic- or inorganic-contaminated sites. Glycolipids and lipopeptides are gaining commercial interest every time more. of Acknowledgements

None.

\section{Conflict of interest}

The author declares no conflict of interest.

\section{References}

1. Banat IM, Makkar RS, Cameotra SS. Potential commercial applications of microbial surfactants. Appl Microbiol Biotechnol. 2000;53(5):495-508.

2. Ron EZ, Rosenberg E, Natural roles of biosurfactants. Environ Microbiol. 2001;3(4):229-236.

3. Karanth NG, Deo PG, Veenanadig NK. Microbial production of biosurfactants and their importance. Curr Sci. 1999;77:116-125.

4. Soberón Chávez G, Lépine F, Déziel E. Production of rhamnolipids by Pseudomonas aeruginosa. Appl Microbiol Biotechnol. 2005;68(6):718725 .

5. Souza EC, Vessoni Penna T, Pinheiro De Souza R. Biosurfactantenhanced hydrocarbon bioremediation: An overview. Int Biodeterior Biodegradation. 2014;89:88-94.

6. Arutchelvi J, Joseph Ch, Doble M. Process optimization for the production of rhamnolipid and formation of biofilm by Pseudomonas aeruginosa CPCL on polypropylene. Biochem Eng J. 2011;56(1-2):37-45.

7. Chrzanowski $Ł$, Dziadas $M$, Ławniczak $€$, et al. Biodegradation of rhamnolipids in liquid cultures: Effect of biosurfactant dissipation on diesel fuel/B20 blend biodegradation efficiency and bacterial community composition. Bioresour Technol. 2012;111:328-335.

8. Cortés Sánchez AJ, Hernández Sánchez H, Jaramillo Flores ME. Biological activity of glycolipids produced by microorganisms: New trends and possible therapeutic alternatives. Microbiol Res. 2013;168(1):22-32.

9. Konishi M, Yoshida Y, Horiuchi J. Efficient production of sophorolipids by Starmerella bombicola using a corncob hydrolysate médium. $J$ Biosci Bioeng. 2015;119(3):317-322.

10. Abbasi H, Noghabi KA, Hamedia MM, et al. Physicochemical characterization of a monorhamnolipid secreted by Pseudomonas aeruginosa MA01 in aqueous media. An experimental and molecular dynamics study. Colloids Surf B Biointerfaces. 2013;101:256-265.

11. Shete AM, Wadhawa G, Banat IM, et al. Mapping of patents on bioemulsifier and biosurfactant: A review. JSIR. 2006;65(2):91-115. 